\title{
Efficient Ray Tracing with Real Weather Data
}

\author{
Pascal Richter* Janna Tinnes* Peter Schwarzbözl ** \\ Amadeus Rong** \\ * RWTH Aachen University, Department of Mathematics, MathCCES, \\ Schinkelstraße 2, 52062 Aachen, Germany (e-mail: \\ richter@mathcces.rwth-aachen.de). \\ ** German Aerospace Center (DLR), Institute for Solar Research, \\ Linder Höhe, 51147 Cologne, Germany.
}

Keywords: Solar energy, Mathematical models, Optimization, Simulation, Sampling, Quadrature, Software Performance

\section{INTRODUCTION}

The optimized layout of a central receiver power plants requires an accurate but fast simulation of the plant operation depending on its design parameters. Ray tracing models are commonly used to calculate the solar flux concentrated by the heliostat field. The computation time for an annual performance simulation is critical for the usage in an optimization procedure.

The main influences on run-time are the spatial (number of rays) and temporal (number of time points) discretization. For the annual simulation, usually weather data from clear sky models is used, e.g. the meteorological radiation model (MRM), see Badescu (2008). Using non-symmetric measured weather data (e.g. from a TMY file) the temporal sample points have to be chosen in a different way.

In this paper, different temporal integration approaches are presented and discussed for the case of measured weather data.

\section{TEMPORAL INTEGRATION}

The annual energy production $E_{y e a r}$ of the solar tower power plant can be computed with the sum over all days $d$ and the integral of the daily power production,

$$
E_{\text {year }}=\sum_{d=1}^{365} \underbrace{\left(\int_{\text {sunrise }}^{\text {sunset }} P(t, d) \mathrm{d} t\right)}_{=: E(D N I(d))},
$$

where $E(D N I(d))$ describes the direct normal irradiation at day $d$. The computation of the annual energy production can be accelerated by reducing the number of samples per day by quadrature rules and reducing the number of days by clustering.

\subsection{Quadrature methods for intraday sampling}

Quadrature methods can be used to approximate the integral of the daily energy $E(D N I(d))$ by using specific sampling points and their according temporal weight.
Because hourly data is provided by the weather files, so far just quadrature methods with a constant time step of one hour are used, e.g. the summed midpoint and summed trapezoidal rule. But to apply quadrature rules with higher order (e.g. Gauss-Legendre quadrature rule) and reduce the number of sampling points, a higher temporal resolution than just hourly constant data is helpful and would further increase the accuracy. It is possible to achieve a higher resolution from the hourly averaged measured data by using data reconstruction, see Fig. 2.1.

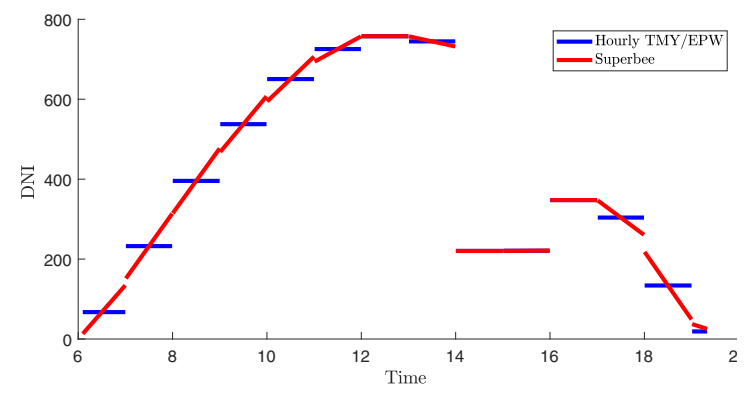

Fig. 1. Reconstruction of the hourly averaged measured DNI data (blue) using the conservative and TVD superbee limiter (red).

DNI value at day $d$ around the time $t_{i}$ changes as follows:

$$
\begin{aligned}
D N I(t, d) & =D N I\left(t_{i}, d\right)+\sigma\left(t_{i}\right) \cdot\left(t-t_{i}\right), \\
t & \in\left[t_{i}-\frac{\Delta t}{2}, t_{i}+\frac{\Delta t}{2}\right],
\end{aligned}
$$

with its originally measured value $D N I\left(t_{i}, d\right)$, and reconstructed slope $\sigma\left(t_{i}\right)$.

Calculating the reconstructed slope with the superbee limiter, which fulfills the required properties of conservativ and TVD, see Leveque (1986), the error between the hourly averaged measured values and the real values can be reduced by $32 \%$ for a cloudy day. For a clear sky, e.g. the data from the MRM model, the error can be reduced by $85 \%$.

With the reconstructed values three different quadraturerules, midpoint, trapezoidal and Gauss-Legendre with a changing number of quadrature points are investigated 
for computing the daily energy, see Fig. 2.1. For Mumbai around 7 sample points per day are needed for a sufficient accuracy.

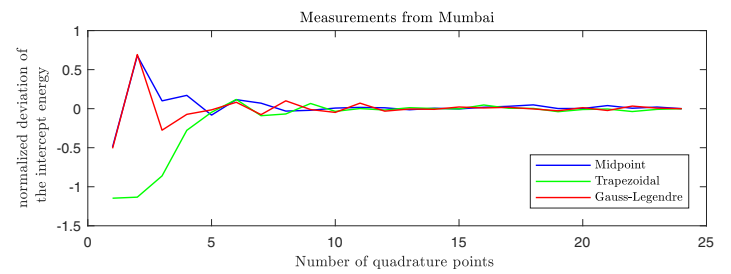

Fig. 2. Comparison of the three quadrature-rules with a changing number of quadrature points for Mumbai.

\subsection{Clustering of days}

Considering the different, day dependent weather conditions an averaged DNI value of the neighboring days can be computed by

$$
D \tilde{N} I\left(d_{k}\right)=\sum_{i=\frac{d_{k-1}+d_{k}}{2}}^{\frac{d_{k}+d_{k+1}}{2}} \frac{2}{d_{k+1}-d_{k-1}} D N I\left(d_{i}\right) .
$$

Using this averaged DNI for the simulated, representing day the annual energy can be computed with the summed trapezoidal-rule

$$
E_{\text {year }} \approx \sum_{k=1}^{m-1} \frac{d_{k+1}-d_{k}}{2}\left(E\left(D \tilde{N} I\left(d_{k+1}\right)\right)+E\left(D \tilde{N} I\left(d_{k}\right)\right)\right) .
$$

Comparing the annual energy computed with the aggregated approach with the usually used constant day sampling, the new approach allows to reduce the number of sample points to only 38 points, see Fig. 2.2.

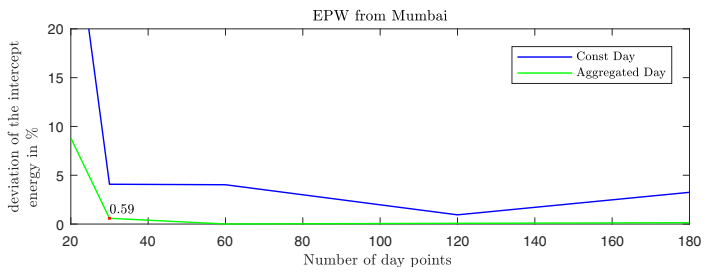

Fig. 3. Comparison of the constant day sampling with the aggregated day sampling approach for a changing number of sample points.

\section{ANGULAR INTEGRATION}

The annual energy production $E_{\text {year }}$ can also be computed by considering the sun path in the domain of the solar angles instead of the time domain. Therefore, the DNI needs to be transformed from the time domain into the angular domain. Then, two-dimensional quadrature rules are used to compute the annual energy, e.g. midpointrule, trapezoidal-rule, Gauss-Legendre quadrature-rule. The underlying quadrature method defines a region in the angular-solar domain. All DNI values of this region are aggregated to one average DNI value, while the number of data points resemble the temporal weight of this region, see Fig 3.

Using the integration in the azimuth-altitude domain the number of sampling points for the computation of the annual energy for Mumbai can be reduced to 18 .
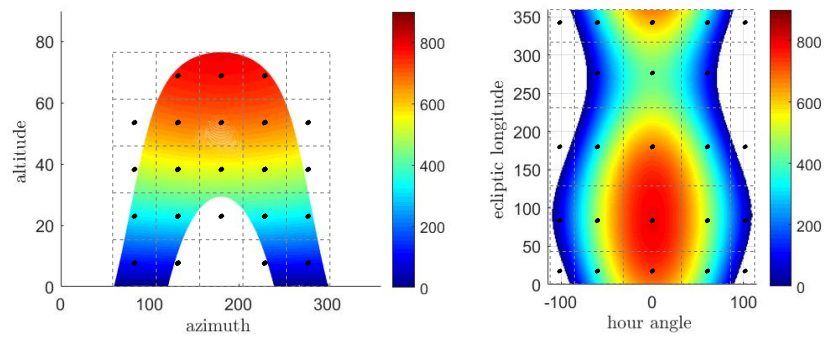

Fig. 4. Integration domain for the transformation to azimuth and altitude with a midpoint grid (left) and to ecliptic longitude hour angle with a Gauss-Legendre grid (right).

A transformation to the hour angle and the ecliptic longitude gives further enhancements, such as the almost rectangular shape of the integration domain, see V. Grigoriev and Blanco (2015). For Mumbai the number of sample points could be reduced further to just 16 sample points per year.

\section{FIELD EFFICIENCY MAP}

Looking at the equation for the power of one heliostat $P_{i}$ with the number of considered losses $n$,

$$
P_{i}(t, d)=A_{i} \cdot I_{\mathrm{DNI}}(t, d) \cdot \sum^{n} \eta_{n, i}(t, d)
$$

the power depends linearly on the intensity of the sun $I_{\text {DNI }}(t, d)$. This allows to uncouple the DNI values and the efficiency of the power plant. A field efficiency map is computed, including the efficiency of the power plant for all possible sun positions, by using e.g polynomial interpolation or spline functions. With this field efficiency map the energy of the power plant can be computed extremely fast by only multiplying the efficiency with the corresponding DNI value.

\section{CONCLUSION}

For industrial performance computations, real measured weather data should be used. For that case, smarter integration methods and a field efficiency map are successfully used, such that the simulation time is strongly reduced.

\section{ACKNOWLEDGEMENTS}

The authors acknowledge the financial support by the Federal Ministry for Economic Affairs and Energy of Germany in the project SolFieOpt (project number 0324039) for the RWTH Aachen University and the project ISAPT (project number 0325655) for the German Aerospace Center (DLR).

\section{REFERENCES}

Badescu, V. (2008). Modeling Solar Radiation at the Earth's Surface. Springer Berlin Heidelberg.

Leveque, R. (1986). Theory and Numerics for Hyperbolic Conservation Laws. Teubner, Stuttgart.

V. Grigoriev, C.C. and Blanco, M. (2015). Fourier sampling of sun path for applications in solar energy. Solar PACES South Africa. 